\title{
A Research Proposition for Using High Definition Video in Emergency Medical Services
}

\begin{abstract}
:
In emergency situations, communication between the ambulance crew and an emergency department in the hospital can be crucial in determining the best decision for a patient's health. Currently, when an ambulance crew reports at an emergency, paramedics use voice communication from scene of emergency to the hospital despite the fact that most modern ambulances will have access to an Internet connection and video equipment. In critical life threatening situations, use of high quality visual images and live video streaming can allow paramedics on the scene of an emergency to take better informed decisions by liaising with expert consultants in the hospital emergency department. This paper proposes the relay of high definition video between the ambulance crew and the hospital using public Internet infrastructure through utilising a virtual path slice controller. The paper also proposes a set of criteria for evaluating the use of video in emergency scenarios taking into account technical, user, application and process requirements together with an overview of the benefits, risks and ethical issues.
\end{abstract}

Keywords: e-health, high definition video communication, emergency, ambulance, evaluation criteria

\section{Introduction}

The National Health Service (NHS) was introduced in 1948 as a publicly funded healthcare system to provide patient care for UK citizens. The NHS is driven by patients' clinical needs rather than by the patients' ability to pay and it was created with the aim that citizens could access healthcare services regardless of their economic status. It provides various services such as emergency and urgent care, general practitioners, hospitals, pharmacies and dental services. The NHS is a large scale [1] and complex organisation [2] that serves over 50 million citizens [3]. For example, the NHS performs over 300 million consultations and over 4 million emergency hospital admissions in a year [1]. Considering the scale of the NHS, one of the most significant challenges is to make healthcare affordable for all citizens [4]. The pressure is even higher now, as the current economic climate has led to numerous budget cuts in the NHS. Moreover, in the UK in particular, and Europe in general, is facing an aging population and increases in healthcare costs, which is compounded by insufficient medical staff [5]. To reduce NHS costs and increase its efficiency, Information and Communication Technology (ICT) is seen as a critical element [3].

NHS spending on ICT (computers and services) increased fast [6], and from an organisation that did not have any computers in 1960 it is now one of the most ICT intense organisations [7]. Several online services have been introduced, including patient administration systems in the 1960s, followed by laboratory and radiology systems in 1970s, hospital information support systems and resource management system in 1980s and electronic health records in 1990s [3]. In 2002, the NHS National Programme for IT (NPfIT) was established, as part of the Health Department. It was driven by an NHS modernisation agenda and its aim was to improve the quality of healthcare through the use of ICT. Among the services that NPfIT delivered, one can count: digitised patient and GP records, a national electronic system through which the patients can choose to book an appointment, an electronic prescription system, a picture archiving and communication systems that allows pictures such as x-rays to be stored in an electronic format etc. These services aim at being convenient and easy to use but also to improve the NHS and have a more effective system of managing patient care. For example, the choose and book system was aimed at making the appointment booking easier and time convenient for both medical staff and patients, and hence reducing wasted time as a result of patients not being able to show up. It also aimed at increasing the patients' freedom in choosing the hospital, consultant, date and time of their medical appointment [3]. Although the idea of involving patients in decision making, and giving them freedom of choice was well received, the patients and medical staff were not involved during the design stages of the service development, leading to problems in system acceptance [3]. 
Review of modernisation in the NHS suggests that although some of the proposed services have been successful, the introduction of ICT through the NPfIT programme did not happen without challenges. The programme has been criticised by scholars and medical staff alike for failure to deliver promised benefits [1]. Moreover, from a patient care perspective, the NHS is criticised for having long patient waiting lists, and an acute shortage of staff [8]. The current economic climate puts further strains on NHS as the government seeks to reduce the resources allocated to healthcare [9]. This may also have an effect on the quality of health care provided to patients. In this paper, we argue that in a climate of economic and resource constraints it is still possible to improve the services offered by the emergency medical services in a cost effective manner. This is, through the use of public Internet infrastructure to transmit high definition video between the ambulance paramedics who call at the scene of an emergency and the consultation point (usually a hospital). As currently the communication between the ambulance and the hospital is done through a radio or mobile voice call [10], adding video could allow the consultation point to have a better view of the emergency situation, hence facilitating a more informed decision regarding the patient condition [47]. This can lead to the appropriate intervention for the patient which may increase his/her chances of recovery and possible cost savings for the NHS (i.e. reducing the time the patient needs to be hospitalised) [49][50][33]. In this context, this paper discusses the potential of using high definition video between emergency scenes or an ambulance and the consultation point.

In order to examine the above, the paper is structured as follow. The next section discusses the use of ICT in NHS services. This is followed by describing how high definition video-to-video communication can be used in emergency scenarios. A framework for evaluating the usage of high definition video in emergency services is presented next. Afterwards, this article discusses the benefits, risks, and ethical issues that rise from the use of video in emergency scenarios. Finally, the paper summarises the key arguments presented in the study and points towards future research.

\section{Background}

In the last few years, electronic healthcare (or e-Health) has been increasingly used as an integral part of both national and international policies to facilitate improved healthcare access and to increase the efficiency, effectiveness and quality of healthcare services as well as a means to reduce costs [13][14][15][16]. e-Health, is defined by the World Health Organisation as "the combined use of electronic communication and information technology in the health sector" [11]. E-Health is seen as a means "to provide better citizen-centred care, as well as lowering the costs and supporting interoperability across national boundaries, facilitating patient mobility and safety" [12]. E-Health adoption is important especially in the current economic climate, where there is immense pressure to reduce costs and transform healthcare. In this context, ICT is having a major role in achieving these aims [9][17]. Several e-Health applications such as Electronic Health Records (EHRs) [18][19], picture archiving and communication system [20], tele-medicine [20], have been used in healthcare with the aim of improving medical staff decision making, increasing the quality of services offered to patients [21] and improving the access to information [22].

In the UK, NPfIT is the main programme under which ICT was introduced in the NHS. The aim of the programme is to deliver the following e-Health services: (1) NHS care records service, (2) electronic appointment booking, (3) picture archiving and communications system (PACs), (4) electronic transmission of prescription, and (5) IT infrastructure and network. The programme was launched in 2002 and was abolished in 2011 [23]. Despite the efforts taken to follow best management practices, there were shortcomings in the usage of IT within the NHS [24]. For example, one of the policies was the introduction of electronic health records for over 50 million citizens [18]. This proved not to be as successful as expected. Many early adopters were frustrated with the non-transparent way in which the services were implemented. This had as a consequence, a reticence felt by later users to use the system [1]. Many of the problems experienced with the services offered by NPfIT programmes were mostly felt by the early adopters [25], and it could be sensible to see this programme as a learning experience [26]. Moreover, progress has been made on other services; such as picture archiving and communication systems and the complexities of scaling electronic health record adoption are better understood now [18][27]. 
Despite the initial challenges, e-Health is changing healthcare use in many countries around the world, including the UK [28]. Although the NPfIT programme was abolished by the present government in the UK, the consultations performed by the Department of Health in the UK has highlighted concerns regarding the need for integration of ICT across health, followed by the necessity to consult beforehand with healthcare professionals [29]. The last issue is especially important, as in the NHS, e-Health adoption has been poor for both practitioners and patients with some of the introduced e-services meeting resistance from key stakeholders from the beginning [18]. For example, patients were not involved in the early stages of decision making and clinicians expressed concerns about patient privacy and were not convinced about the benefits added by the given eservices [18].

Problems with e-Health adoption have been reported also outside the UK [30][31]. This, despite the fact that eHealth has been shown to provide better data quality, availability, accessibility, connectivity, exchange, and sharing [15][16]. In USA, it is estimated that with a minimum level of automation, a reduction of 50-80\% in life loss could be achieved [32]. Prior research has identified several challenges with the current use of e-Health with regards to the communication infrastructure [33][34], adoption and use of e-Health services [15][18] and privacy, security and confidentiality [9], the necessity of the system to be user centred [35] and the necessity to explore new technology [36].

This paper focuses on a particular type of e-health service, the application of video technology for emergency services. Although use of video in health emergency services has been recommended it is an uncommon practice, and communication has been traditionally done through voice call [10][33]. In emergency services, ehealth has been mostly used in delivering the vital signs and assuring voice communication between the ambulance and hospital [33]. Several studies have been successful in using mobile devices to transmit patient ECGs between the pre-hospital and the specialist hospital, and have been proven especially beneficial for patients suffering of acute coronary syndrome [37][38][39].

Technical problems have also been reported in several of these studies. For example, Adams et al., [37] reported failures in ECG transmission for $44 \%$ of the cases, whereas a smaller failure rate (14\%) is reported by Terkelsen et al., [40]. When video has been used, Kwak [41] reports a failure of $16 \%$ and Bergrath et al., [33] reports 3\% failure in video transmission, highlighting the importance of assuring reliable connectivity between the ambulance and the contact point. In this research, we explore the usage of high definition video between the ambulance paramedic and the consultation point (i.e. the hospital emergency room) over the public infrastructure or Internet. Although video communication between the ambulance crew and the hospital has been previously reported in the literature [33][34][44][45] the evaluation of these systems has been observed mostly through simulations [33]. On top of the failures in delivering high quality video that leads to a total unusable application [33][41] previous studies report various problems that appear with video delivery over wireless networks where delays and data loss has led to poor video quality and unusable systems [33][34][44][45]. For example, if an electrocardiogram (ECG) is to be transmitted, transmission errors due to loss of data could lead to an erroneous ECG and hence misjudgements with regards to the patient state [44]. This is also the case when a glaucoma diagnosis is necessary; a good picture of the patient eye is necessary so that this is not confusing for the specialist [46]. This research proposes to address such problems reported in previous cases of video use in the healthcare context through the use of uninterrupted, high definition video over the Internet.

\section{Using Video to Video Technology in Emergency Services in the NHS: A Research Proposition}

Although various e-health services already exist within NHS, in this paper we focus on emergency services. In this context, we looked at how high definition video conferencing can be used to improve existing services by providing a richer communication between ambulance doctors and the contact point (usually a specialist doctor from the hospital) and how to alleviate some of the problems faced by video delivery over the current public network infrastructure. 
Uninterrupted communication between the ambulance crew on the ground and the contact point is necessary when the ambulance crew on the scene needs to communicate to a specialist either asking for advice regarding the patient treatment or to decide the best hospital to which the patient can be sent. Currently this is done through either radio or a mobile phone call [10]. Although useful, the communication suffers the drawbacks that voice has: the specialist cannot be provided with more information than the information that the ambulance crew is describing. For example, a doctor from the hospital cannot see the patient or the results of the analysis performed in place (i.e. real time biosignals such as ECG, blood pressure, blood glucose) or the patient. Therefore, the view of the situation is limited, and not all information is available to provide an informed decision. In emergency situations having this information can be critical for patient wellbeing. Consequently, having video together with voice between the ambulance and the hospital (Figure 1) will provide the hospital with more information regarding the emergency situation with which the ambulance crew is dealing with. The paramedic could have a camera installed on the ambulance and a mobile camera attached to his equipment to stream live video to the contact point providing a better overview of the situation.

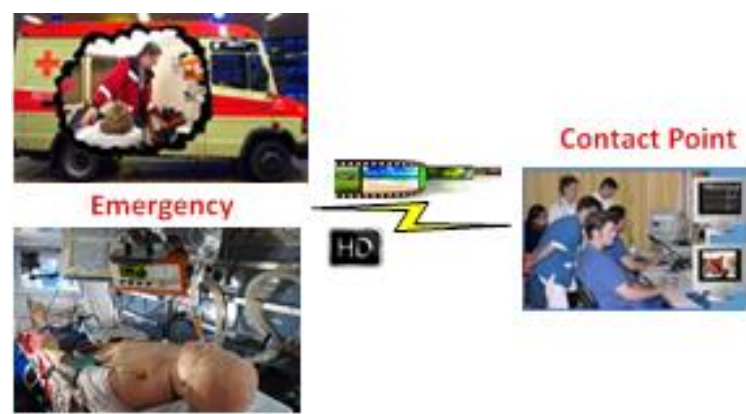

Figure 1 Video Communication between an Ambulance and Hospital

Video communication could help both in speeding [38][42][40] and providing a more informed decision regarding the patient treatment [33], advice given to the ambulance crew on how to treat the patient in case of advanced diagnosis and when immediate treatment is required, and deciding the appropriate delivery of the patient to the adequate treatment unit [47] particularly in situations where time is critical such as in myocardial infractions [50]. For example, in the case of a trauma patient with entrapment it is useful to communicate to the hospital the environment in which the patient is. In this case, the ambulance crew may require specialist advice regarding the optimal treatment and the need for speedy evacuation. It could also facilitate decision making regarding the need for another team to be dispatched at the scene, the appropriate unit in which the patient(s) have to be delivered and the necessary preparation the unit has to make to appropriately respond to the incoming patient(s) [47]. It could also optimise resources by deciding which patient(s) requires immediate attention and needs to be fast tracked for treatment, but also avoiding delivering to the hospital patients that do not require treatment or could be treated on scene or elsewhere (i.e. in a primary care setting). Another scenario in which video will add value is for the patients for which immediate attention is required, especially when delivering patients to a healthcare unit requires a long time (either due to long distances [44] or traffic congestion [45]). This may reduce the possibility of providing the patient care within the "golden hours". The golden hour is defined as "the first crucial hour after injury during which early interventions can significantly lower rates of morbidity and mortality" [44]. There are also situations in which drugs work only if delivered within a certain interval from when the symptoms appear (e.g. clot-busting drugs).

Previous studies performed on video between an ambulance and hospitals reported various difficulties encountered when delivering video over Internet such as problems with data loss and as a consequence leading to a poor video quality [33][34]. This research argues that such problems can be alleviated to deliver high definition video on public Internet infrastructure through the use of a Virtual Path Slice (VPS) controller (Figure 2) that can manage bandwidth by avoiding interference from unwanted traffic and therefore assuring that any loss of delivered data and delays are reduced [47][42]. We posit that the VPS controller can be used on the existing Internet infrastructure and it can lead to improvements in the quality of the video delivery. The ability 
to see a good quality video picture may be critical in these situations when the speed of decision making is of paramount importance for patient health being.

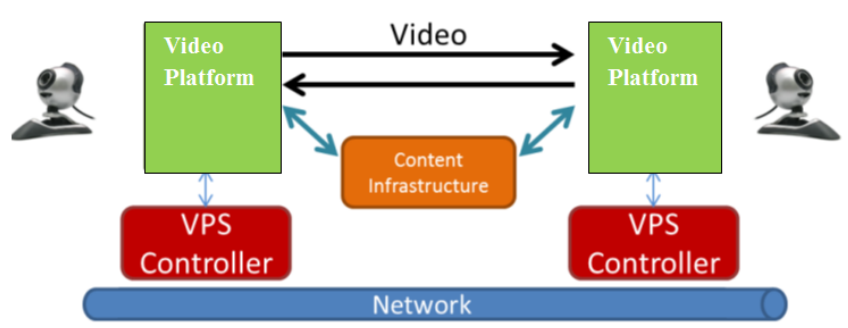

Figure 2 Video Platform with the Use of VPS Controller [42]

While it is not our intension to discuss the technical characteristics and functions of the VPS controller in this paper, essentially it is an application that allows network bandwidth to be controlled in a way where a virtual path is created on the public Internet to allow traffic (data, voice and video) to flow freely without losing quality. This enables by ensuring that the signals are not interrupted by other external Internet traffic when connection between two points of contact are established. In this respect, the VPS controller allows the creation of a path that is similar to a virtual private network to guarantee transmission of high quality live video images between two points of contact. As such, the VPS controller will allow the transmission of high definition video from an ambulance crew at a scene of an emergency to a base hospital emergency room where doctors can monitor and direct appropriate action.

\section{A Framework for the Evaluating Usage of High Definition Video in Emergency Services}

Provisioning of high definition video communication in emergency services over the current existing network infrastructure requires technical changes, but could result in existing service delivery procedures and related outcome being improved. Nonetheless, this requires careful consideration of the technical, application, process and user level criteria and how they are enhanced compared to existing methods of service delivery. However, due to the innovative nature of using high definition video conferencing, there does not exist an established way of evaluating the use of high definition video in emergency context. Therefore, we draw from previous literature in order to propose a set of evaluation criteria for high definition video communication between the ambulance paramedics and hospital. The evaluation of such applications has to take into account the following dimensions: technical, user, application, and process requirements (Figure 3). For each dimension, the evaluation criteria are identified.

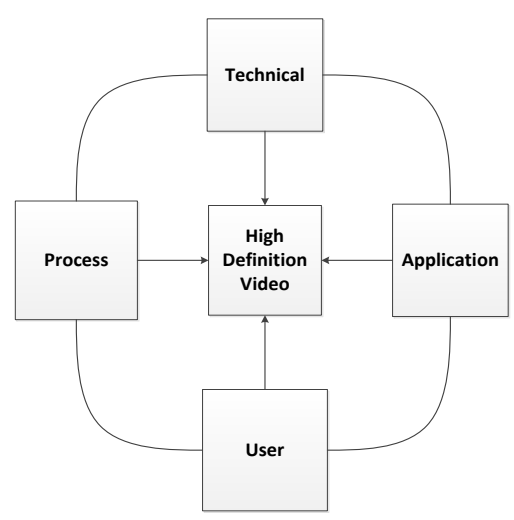

Figure 3 Framework for Evaluating Video Services in Emergency Scenario 
Technical Requirements: are related mostly to network parameter requirements for ensuring good connectivity, scalability and flexibility, fault tolerance and reliability, and security and privacy (Table 1). Provisioning a good connectivity is imperative if uninterrupted, high definition video is to be transmitted between two points, such as an ambulance and a hospital; and its importance has been stated in previous other pilots studies of using video in emergency situations [33][34][44][45]. Due to the number of patients using NHS serves, and any other public emergency service, scalability and flexibility is an important parameter to be considered. The importance of having a fault tolerant and reliable system has been probably best highlighted during the usage of London Ambulance Service's Computer Aided Dispatch System, whose failure has led to ambulances coming too late and newspapers claiming that 20-30 people have lost their life as a result [43]. In addition, security of information and privacy are elements that have been highlighted by both clinicians and patients during the NPfIT programme [9].

Table 1 Technical Requirements

\begin{tabular}{|l|l|}
\hline Issues & Description \\
\hline Connectivity & $\begin{array}{l}\text { Connection between the paramedic and hospital should be fast and error } \\
\text { free and should provide a seamless end to end high definition video } \\
\text { communication. }\end{array}$ \\
\hline Scalability and flexibility & $\begin{array}{l}\text { Connection between the paramedic and hospital should support multiple } \\
\text { concurrent connections. }\end{array}$ \\
\hline Fault tolerance and reliability & $\begin{array}{l}\text { The video service platform should successfully recover from an error and } \\
\text { be available to the user and perform the correct operations. }\end{array}$ \\
\hline Security and Privacy & $\begin{array}{l}\text { Video images and data should be securely transmitted over fixed and } \\
\text { wireless networks. }\end{array}$ \\
\hline
\end{tabular}

User Requirements: are related to the actual utilisation of the video communication by the involved users and the respective level of User Experience (Table 2). The evaluation criteria are built around:

- Usability/Ease of Use: the service shall be easy to use by different medical staff (i.e. emergency doctor, nurse) [9].

- Satisfaction: favourableness with respect to the system and the mechanics of interaction.

- Reliability: reflects the user point of view regarding reliability aspects of the video provision.

Table 2 User Requirements

\begin{tabular}{|l|l|}
\hline Issues & Description \\
\hline Usability/Ease of Use & $\begin{array}{l}\text { The video service platform should be easy to use by both the ambulance } \\
\text { crew and the contact point. }\end{array}$ \\
\hline Satisfaction & $\begin{array}{l}\text { The user (i.e. paramedic, nurse, and specialist) should have a good overall } \\
\text { opinion of the system. }\end{array}$ \\
\hline Reliability & $\begin{array}{l}\text { The video service platform or system should always and consistently show } \\
\text { high definition and uninterrupted images and data for the given use case } \\
\text { context. }\end{array}$ \\
\hline
\end{tabular}

Application Requirements: are related to the requirements the application has, such as variability of the device, support for codecs, performance and hardware resources necessary for running the application (Table 3).

Table 3 Application Requirements

\begin{tabular}{|l|l|}
\hline Issues & Description \\
\hline
\end{tabular}




\begin{tabular}{|l|l|}
\hline Variability of the device & $\begin{array}{l}\text { The video service platform should be easily accessible from different } \\
\text { devices (i.e. P.C., mobile phone). }\end{array}$ \\
\hline Support for codecs & The platform should support different video codecs. \\
\hline Hardware resources & The platform should not require extensive resource usage. \\
\hline
\end{tabular}

Process Requirements: are related to the end-to-end process tailored to the various video provision scenarios. There are several evaluation criteria that can be taken into account at this stage such as: diagnostic time, survival rate, number of days spends by the patient in the hospital, duration time to reach the appropriate hospital. These could be compared with the same results obtained for the communication done by placing a call instead of using video, but also through the usage of the video system without provisioning for a high definition video.

Table 4 Process Requirements

\begin{tabular}{|l|l|}
\hline Issues & Description \\
\hline Diagnostic time & $\begin{array}{l}\text { The usage of video communication between the ambulance and contact } \\
\text { point (i.e. hospital) should reduce the diagnostic time, compared with the } \\
\text { results obtained when a called was placed. }\end{array}$ \\
\hline Survival rate & $\begin{array}{l}\text { The usage of video communication between the ambulance and contact } \\
\text { point (i.e. hospital) should improve survival rate compared with the results } \\
\text { obtained when a called was placed. }\end{array}$ \\
\hline $\begin{array}{l}\text { Number of days spends by } \\
\text { the patient in the hospital }\end{array}$ & $\begin{array}{l}\text { The usage of video communication between the ambulance and contact } \\
\text { point (i.e. hospital) should reduce the average number of days the patients } \\
\text { spend in the hospital. }\end{array}$ \\
\hline Travelling time & $\begin{array}{l}\text { The usage of video communication between the ambulance and contact } \\
\text { point (i.e. hospital) should reduce the time needed for the patient to reach } \\
\text { the hospital. }\end{array}$ \\
\hline
\end{tabular}

\section{Discussion}

As we highlighted in the previous section, facilitating high definition video between the ambulance and a contact point may be of paramount importance in patient care in emergency situations. However, as it is usually the case in e-Health the advantages gained by using ICT in healthcare leads to many intangible benefits [48]. It is difficult to put a price on the improved communication between the ambulance and the contact point (e.g. specialist doctor) and the consequences it has on a patient's wellbeing. It has been shown that in certain situations (e.g. myocardial infarction) rapid transfer of the patients to the appropriate hospital leads not only to economic savings but also improvements in patient safety by avoiding further complications [49]. Moreover, delivering preliminary information to the hospital in emergency situations has been shown to reduce treatment delays in the case of patients with acute ST-elevation myocardial infraction [50]. Being able to transmit ECGs to a specialist to remotely interpret them leads also to a faster treatment time for the patients [38] [40][42]. We posit that the advantages gained by the video communication (i.e. more informed decision making leading to an increase in diagnostic accuracy [33], proper management of the patients and medical staff) will improve the quality of the patient treatment that will ensure faster patient recovery, earlier detection of the health problem, lowering of the complications, prevent unnecessary losses of life, prevent and minimise trauma in emergency and accident cases. These results would also lead to savings in tax payers' money as they will reduce the treatment time hence the time spent in the hospital and the cost of medication. In addition, medical resources will be more effectively used (for example by avoiding an unnecessary trip to deliver the patient to a hospital that does not have at the moment resources that the patient requires for the treatment) and it will also prevent cases of litigation due to miss-diagnosis. 
It is also important to recognize that although both the improvement in quality of healthcare and cost reductions is possible, there are also negative cost consequences and risks associated. The costs will include the cost of equipment necessary for video communication. However, as the provisioning of the high definition video communication is done on existing Internet infrastructure, the only cost will be the deployment of a VPS controller and the cost for video transmissions. Yet, there also several risk factors associated with the proposition to use video in emergency healthcare, these include: technical network failures, as well as clinicians and patient concerns regarding "ease of use, data integrity, confidentiality and patient safety" [9]. Technical problems due to network failures can lead to unavailability of the video communication or poor video connectivity and problems with the system design (i.e. the equipment being hard to manipulate hence leading to delays in using it). These risks can be mitigated by allowing video communication to be offered as a complementary service, while relying on voice communication if problems occur. Also, intensive testing before system deployment should be done to identify and solve the problems in network connectivity and usability of the equipment, to avoid frustration of the adopters and reticence in the later users, as it was the case of services offered in the NPfIT programme [1]. This research also provides a step forward in addressing these problems through the use of a VPS controller that should alleviate some of the technical problems the current Internet infrastructure has. Problems with data integrity and confidentiality can be mitigated by using an encrypted connection or NHS ICT network that is a private network designed to ensure confidentiality [51]. As the video communication between the ambulance and contact point is only streamed and no recording is made, no action will be required regarding data storage. Moreover, usage of video in this scenario does not pose any danger to patient safety.

Another important risk to consider is that the introduction of this new technology may not be accepted by the stakeholders (e.g. doctors, hospital administration). This risk can be mitigated by involving the stakeholders in the early stages of decision making and assessing the short and long-term factors that would make this service successful, but also by learning from challenges that previous e-Health projects encountered.

From an ethical perspective, since the video communication system is an add-on approach to the currently existing setting and modalities of communication between the provider of the health/medical service, from one side, and the recipient, from the other, and there is no active treatment by drugs or other new medical/clinical treatments involved, ethical issues, or such that could not be addressed as inherent, do not exist. Other ethical issues such as involving patients during the evaluation of the service should be properly addressed to oversee and safeguard adherence to existing ethical standards for medical/clinical research or IT research in a medical/clinical environment.

\section{Conclusion}

e-Health is aimed at improving the efficiency, effectiveness, and transparency of health services. Despite the advances reached in previous years and improvements in the Internet infrastructure, communication between the ambulance services and hospitals in the UK is still done through voice (either radio or mobile) call. This article has presented how the ambulance service could be improved by providing high definition video between the ambulance crew and the hospital staff (i.e. emergency doctor, specialist). We presented the benefits associated with using such a system and we posit that it would improve the quality of healthcare by reducing its costs and improving decision making. This paper also discussed the risks associated with the adoption of a video based system in emergency care. A set of criteria for evaluating the usage of high definition video in emergency services was also presented outlining technical, user, application and process related issues. These evaluation criteria can be used as a guideline in implementing and evaluating video in emergency services. The discussions offered and arguments presented in this paper are based on a research project that is currently being undertaken by the authors as part of a consortium that is funded by the European Commission. This project is funded under the ICT PSP program of the EC and is entitled LiveCity. The project commenced in May 2012 and is currently developing the infrastructure for testing the use of Live Video in Emergency Scenarios as propositioned in the paper. The actual pilot implementations are planned in Spring/Summer 2013 and future research will explore, evaluate and report the use of high definition video communication both through real case scenarios. From a 
practical perspective this paper offers policy makers a method of improving emergency services and a theoretical framework to evaluate the impact of high definition video usage in emergency services.

\section{Acknowledgements}

The authors wish to acknowledge the contributions made to this article by the LiveCity consortium of partners and the European commission.

\section{References}

[1] Granger R. The NPfIT in the NHS in England, [Internet] 2004. [cited 2013 Jan. 15]; Available from: www.connectingforhealth.nhs.uk.

[2] Mohan J. Planning, Markets and Hospitals 2002, London: Routledge.

[3] Currie WL, Guah MW. Conflicting institutional logics: a national programme for IT in the organisational field of healthcare. Journal of Information Technology 2007;22(3):235-247.

[4] Pollock A. NHS plc: The Privatisation of our Healthcare 2005, London: Verso.

[5] Pinto R, Baracsi M. Creating an environment for innovative start-ups in healthcare. Health Policy and Technology 2012;1(4):187-192.

[6] National Audit Office. The National Programme for IT in the NHS 200416 June, London: The Stationary Office.

[7] Brennan S. The NHS IT Project 2005, Radcliffe Publishing Ltd, Oxon.

[8] Robinson PG. Modelling the dental workforce supply in England [PhD thesis]. University of Sheffield; 2011.

[9] Currie WL. TEMPEST: An integrative model for health technology assessment. Health Policy and Technology 2012;1(1):35-49.

[10] Patton GG, Thakore S. Reducing inappropriate emergency department attendances - a review of ambulance service attendances at a regional teaching hospital in Scotland. Emergency Medicine Journal. 2012. Available from: http://emj.bmj.com/content/early/2012/07/15/emermed-2012-201116.abstract

[11] World Health Organisation. [Internet] Available from: http://www.who.int/topics/ehealth/en/

[12] Together for health: a strategic approach for EU 2008-2013 [Internet] 2008. [cited 2013 Jan. 3]; Commission health strategy (2008-2013) COM (2007) 630 final. Available from: http://ec.europa.eu/health/ph_overview/Documents/strategy_wp_en.pdf

[13] Charles BL. Telemedicine can lower costs and improve access. Healthcare Financial Management 2000;54(4): 66.

[14] Porter ME, Teisberg EO. Redefining health care: creating value-based competition on results. USA: Harvard Business School Press; 2006.

[15] Stefanou C, Revanoglou A. ERP integration in a healthcare environment: A case study. Journal of Enterprise Information Management 2006;19(1):115-130.

[16] Trimmer K, Pumphrey DL. Wiggins CERP implementation in rural healthcare. Journal of Management in Medicine 2002;16(2-3):113-132.

[17] Martin-Moreno JM, Anttila A, von Karsa L, Alfonso-Sanchez JL, Gorgojo L. Cancer screening and health system resilience: Keys to protecting and bolstering preventive services during a financial crisis. European Journal of Cancer 2012;48(14):2212-2218

[18]Currie WL. Evaluating the governance structure for public sector IT: The UK National Programme in the Health Service. Evaluating Information Systems 2012;p.199-217.

[19]Lafky D, Tulu B, Horan T. Information systems and Health Care X: A user driven approach to personal health records. Comm. of the Association for IS 2006;17:1028-1041.

[20] Menachemi N, Burke DE, Ayers DJ. Factors affecting the adoption of telemedicine-A multiple adopter perspective. Journal of Medical Systems 2004;28(6):617-632.

[21] Mantzana V, Themistocleous M, Morabito V, Soulioutis K. Evaluating actors and factors associated with healthcare information systems. Evaluating Information Systems 2008;p.179-198.

[22] Lenz R, Kuhn KA. Towards a continuous evolution and adaptation of information systems in healthcare. International Journal of Medical Informatics 2004;73(1):75-90. 
[23]NPfIT Review. Major Projects Authority Program Assessment Review Of The National Program For IT. Cabinet Office [Internet] 2011. [cited 2013 Jan. 3]; Available from: http://www.cabinetoffice.gov.uk/sites/default/files/resources/mpa-review-nhs-it.pdf

[24] Currie WL. Institutional isomorphism and change: the national programme for IT-10 years on. Journal of Information Technology 2012; 27(3):236-248.

[25] Takian A, Cornford T. NHS information: Revolution or evolution?. Health Policy and Technology 2012;1(4): 193-198.

[26] Haughom JL. Implementation of an electronic health record. BMJ 2011;p.343

[27] Cresswell K, Aziz S. The NHS Care Record Service (NHS CRS): recommendations from the literature on successful implementation and adoption. Informatics in primary care 2009;17(3):153-160.

[28] Atherton H, Azeem M. An information revolution: time for the NHS to step up to the challenge. JRSM 104.6 2011: 228-230.

[29] Liberating the NHS: an information revolution. A summary of consultation responses. London: Department of Health [Internet] 2011. [cited 2013 Jan. 3]; Available from: http://www.dh.gov.uk/prod_consum_dh/groups/dh_digitalassets/documents/digitalasset/dh_129580.pdf

[30] Westrup C. What' s in Information Technology. Asian Institute of Technology , Bangkok; 1998.

[31] Heeks R, Mundy D, Salazar A. Why Health Care Information Systems Succeed or Fail. Institute for Development Policy and Management [Internet] 1999. [cited 2013 Jan. 3]; Available from: http://www.man.ac.uk/idpm/idpm_dp.htm\#isps_wp.

[32] Sutherland J, Willem J. Enterprise application integration and complex adaptive systems. Communications of the ACM 2002; 45(10):59-64.

[33] Bergrath S, Rörtgen D, Rossaint R, Beckers SK, Fischermann H, Brokmann JC, et al. Technical and organisational feasibility of a multifunctional telemedicine system in an emergency medical service-an observational study. Journal of Telemedicine and Telecare 2011;17(7): 371-377.

[34] Cheon G, Shin IH, Jung, MY, Kim, HC. Implementation of a real-time multi-channel gateway server in ubiquitous integrated biotelemetry system for emergency care (UIBSEC). In Proceedings of the IEEE Annual International Conference of the Engineering in Medicine and Biology Society; 2009;p.207-5210.

[35] Lichtner V, Petrakaki D, Hibberd R, Venters W, Cornford T, Barber N. Mapping stakeholders for system evaluation - the case of the Electronic Prescription Service in England. In: Safran C, Reti S, Marin H, editors. Medinfo 2010-Proceedings of the 13th World Congress on Medical Informatics, Cape Town, South Africa: IOS Press; 2010;p.1221-25.

[36] Coiera E. Why system inertia makes health reform so difficult. BMJ 2011:p.342.

[37] Adams GL. et al. Effectiveness of prehospital wireless transmission of electrocardiograms to a cardiologist via hand-held device for patients with acute myocardial infarction (from the Timely Intervention in Myocardial Emergency, NorthEast Experience [TIME-NE]). The American journal of cardiology 2006;98(9):1160-1164.

[38] Sejersten M. et al. Effect on treatment delay of prehospital teletransmission of 12-lead electrocardiogram to a cardiologist for immediate triage and direct referral of patients with ST-segment elevation acute myocardial infarction to primary percutaneous coronary intervention. American Journal of Cardiology 101.7 (2008): 941-946.

[39] Ting H.H. et al. Implementation and integration of prehospital ECGs into systems of care for acute coronary syndrome A scientific statement from the American Heart Association Interdisciplinary Council on quality of care and outcomes research, emergency cardiovascular care committee, council on cardiovascular nursing, and council on clinical cardiology. Circulation 2008;118(10):1066-1079.

[40] Terkelsen C.J. et al. Telemedicine used for remote prehospital diagnosing in patients suspected of acute myocardial infarction. Journal of internal medicine 2002;252(5):412-420.

[41] Kwak MJ. et al. Real-time medical control using a wireless audio-video transmission device in a prehospital emergency service in Korea. Journal of Telemedicine and Telecare 2009;15(8):404-408.

[42] Chochliouros I.P. Description and thematic context of the LiveCity project. Conference of Telecommunication, Media and Internet Techno-Economics. Workshop on Network Operators View on Future Networks Landscape: Will it be Cloudy with a Chance of Data Rain? [Internet] 2012. [cited 2013 
Feb. $\quad 4] ; \quad$ Available $\quad$ http://www.ctteconference.org/files/2011/CTTE_OperatorsWorkshop_Chochliouros_LiveCity.pdf

[43] Beynon-Davies P. Information systems failure and risk assessment: the case of the London Ambulance Service Computer Aided Despatch System. European Conference on Information Systems. 1995;34:11531170.

[44] Chu Y, Ganz A. A mobile teletrauma system for rural trauma care. In Proceedings of the IEEE Annual International Conference of the Engineering in Medicine and Biology Society 2004;2:3282-3285.

[45] Banitsas K, Tachakra S, Stefanidis E, Boletis K. Using HSPA to improve the telemedical links on a moving In Proceedings of the 30th Annual International Conference of the IEEE Annual International Conference of the Engineering in Medicine and Biology Society 2008;p.739-742.

[46] Stamatelatos M, et al. Video-to-video for e-Health: Use case, concepts and pilot plan. Artificial Intelligence Applications and Innovations (2012):p.311-321.

[47] Weerakkody V, El-Haddadeh R, Molnar A. Examining the Impact of Utilising Video to Video Technology for Delivering Public Sector Services, E-Government Services Design, Adoption, and Evaluation, V. Weerakkody, IGI Global; 2012

[48] Whitten PS, Mair FS, Haycox A, May CR. Williams, TL, Hellmich S. Systematic review of cost effectiveness studies of telemedicine interventions. Bmj 2002;324(7351):1434-1437.

[49] Dewurst MJ, McComb JM. Permanent pacing in patients with complete heart block: the potential safety and cost savings of direct transfer from the ambulance service to a pacing centre. Europace 2012; 14.suppl 4: iv18-iv21.

[50] Scholz KH, Hilgers R, Ahlersmann D, Duwald H, Nitsche R, von Knobelsdorff, G, et al. Contact-toballoon time and door-to-balloon time after initiation of a formalized data feedback in patients with acute ST-elevation myocardial infarction. American Journal of Cardiology 2008;101(1):46-52.

[51] Coates S, Clarke A, Davison G, Patterson V. Tele-EEG in the UK: a report of over 1000 patients. Journal of Telemedicine and Telecare 2012;18(5):243-246. 